\title{
Threshold effects of COVID-19-confirmed cases on change in pollutants changes: evidence from the Chinese top ten cities
}

\author{
Qiang Wang ${ }^{1,2} \cdot$ Xiaowei Wang ${ }^{1,2}$ \\ Received: 16 February 2021 / Accepted: 13 April 2021 / Published online: 20 April 2021 \\ (C) The Author(s), under exclusive licence to Springer-Verlag GmbH Germany, part of Springer Nature 2021
}

\begin{abstract}
A more comprehensive understanding of the impact of the COVID-19 pandemic on changes in pollution could serve us to better deal with the environmental challenges caused by the pandemic. Existing studies mainly focused on the linear impact of the pandemic on the pollutants without considering the impact of other factors. To fill the research gap, the nonlinear relationship between pandemic and pollutants with considering the temperature factor was explored by developing panel threshold regression approach. In the proposed approach, the number of confirmed cases was set as explanatory variable, concentrations of $\mathrm{NO}_{2}$ and PM2.5 were set as explained variables, temperature was used as threshold variable, and other air pollution indicators were used as control variables. The results showed that there is a threshold effect between the changes in confirmed COVID-19 cases and the concentrations of PM2.5 and $\mathrm{NO}_{2}$, confirming the impact of the pandemic on pollutions was nonlinear. The results also show that the negative impact of pandemic on pollution increased when the temperature was rising. This work had theoretical and practical significance. The nonlinear research perspective of this article provided a methodological reference for exploring the relationship between epidemic and pollutant-related variables. Furthermore, this study expanded the scope of application of the threshold panel regression model and enriched the quantitative analysis of epidemics and pollutants.
\end{abstract}

Keywords Air pollutants $\cdot$ Nonlinear relationship $\cdot$ Temperature $\cdot$ COVID-19 $\cdot$ Panel data

\section{Introduction}

To curb the spread of COVID-19, governments of various countries have adopted a series of measures, such as restricting residents from going out, stopping bazaars and

\section{Highlights}

- The panel threshold regression approach was developed.

- Temperature was used as a threshold variable; other air pollution indicators were used as control variables.

- Negative nonlinear impact of pandemic on pollution increased when the temperature was rising.

Responsible Editor: Lotfi Aleya

Qiang Wang

wangqiang7@upc.edu.cn

1 School of Economics and Management, China University of Petroleum (East China), Qingdao 266580, People's Republic of China

2 Institute for Energy Economics and Policy, China University of Petroleum (East China), Qingdao 266580, People's Republic of China gatherings, and suspending work and classes. Transportation and economic activities have dropped significantly, which has caused significant changes in the concentration of air pollutants. China has been facing the dilemma of environmental pollution (Dong and Liu 2020). Scholars were not only concerned about the impact of the weakening of economic activities caused by the control of the epidemic on the changes in pollutants but also the relationship between pollutants and COVID-19.

At present, many scholars have conducted research on the correlation between air quality and the spread of COVID-19. Zhu et al. (2020) collected panel data from 120 cities in China from January 23, 2020, to February 29, 2020, and applied a generalized additive model to study the correlation between six air pollutants and confirmed cases of COVID-19. Pei et al. (2020) used comparative analysis methods and used remote sensing and field observation data to analyze the impact of COVID-19 prevention and control on the atmospheric environment in Beijing, Wuhan, and Guangzhou in China. The results showed that the NO2 concentration showed a downward trend across the country, but the change trend of PM2.5 concentration in different regions was obviously 
different. Lin et al. (2020) evaluated the impact of meteorological parameters and air quality in 29 provinces in China on the spread of COVID-19. The basic reproduction rate was calculated by the maximum likelihood removal method based on the chain binomial model, and the correlation between COVID-19 and atmospheric pollutants or meteorological parameters was estimated through correlation analysis. Saez et al. (2020) studied the effects of long-term exposure to air pollutants on the spread of COVID-19 and the risk of death in Catalonia, Spain. The method used in the article was a hybrid longitudinal ecological design and a generalized linear hybrid model. Domingo et al. (2020) assessed the potential relationship between the impact of air pollutant concentrations on the airborne transmission of SARS-CoV-2 and the severity of the COVID-19 coronavirus infection. Research results showed that long-term exposure to certain air pollutants can lead to more serious coronavirus infections and more difficult recovery. Berman and Ebisu (2020) assessed the air quality during the COVID-19 pandemic in the continental United States from January 8, 2017, to April 21, 2020. The countylevel pollution concentration during the historical period and the new crown pneumonia period was compared. Chen et al. (2020) used the attribution score method to estimate the daily specific cause-of-death mortality that China avoided during mass quarantine due to reduced air pollution. Le et al. (2020) found that during the outbreak of China's new crown pneumonia, air pollution was abnormal, and air pollution was alleviated. Wang et al. (2020) used the community multi-scale air quality model from January 1 to February 12, 2020, to study the changes in PM2.5 in 10 cities in China under the emission reduction scenario after the "blocking the city" of the epidemic. Wu et al. (2020) investigated the number of COVID-19 deaths in more than 3000 counties in the USA and the county-level PM2.5 long-term average, fitted a negative binomial mixed model, and performed more than 68 additional sensitivity analyses. It was found that with an increase of $1 \mu \mathrm{g} / \mathrm{m}^{3} \mathrm{PM} 2.5$, the COVID-19 mortality rate increased by $8 \%$. He et al. (2020) inferred the short-term impact of COVID19 on air pollution in Chinese cities and used the difference model in difference to compare cities with and without lockdown policies. It was found that the city blockade had made a considerable improvement in air quality. Fattorini and Regoli (2020) collected actual COVID-19 data and corresponding long-term air quality assessments in various Italian provinces and elaborated and tested the interaction between them. Zhang et al. (2020) used the data of newly confirmed cases in 219 cities in China from January 24 to February 29, 2020 , to study the impact of weather conditions and air pollution as co-factors on the spread of COVID-19. Contini and Costabile (2020) found that exposure to air pollution can increase vulnerability and adversely affect the prognosis of COVID-19 patients. Shehzad et al. (2020) discussed the effectiveness of COVID-19 on air pollution in Indian territory from January to April 2020. By implementing an artificial neural network model, Magazzino et al. (2020) estimated a precise point in the neural transmission from PM10 and PM2.5 to the number of deaths due to COVID-19 in the three cities of Paris, Lyon, and Marseille in France. This point would describe the predictable concentration of particulate matter, which would lead to an increase in the number of deaths due to COVID-19. Singh et al. (2020) estimated the changes in six standard air pollutants during the national lockdown in India caused by the COVID-19 pandemic. Comunian et al. (2020) analyzed the role of Italian particulate matter in the spread and increase of COVID-19 morbidity and mortality. Gupta et al. (2020) used statistical methods such as analysis of variance and regression models to analyze the relevant data of nine Asian cities and found that air pollution is a basic and hidden factor that increases the global burden of COVID-19-related deaths. Copat et al. (2020) systematically reviewed the literature on the relationship between some atmospheric pollutants (PM2.5, PM10, NO2) and the spread and mortality of COVID19. Cole et al. (2020b) estimated the relationship between longterm air pollution exposure and COVID-19 in 355 municipalities in the Netherlands. Hendryx and Luo (2020) tested the relationship between long-term exposure to air pollutants, possible pollution sources, and the prevalence and mortality of COVID-19 in the USA as of May 31, 2020, through linear multiple regression analysis with mixed models. Adams (2020) assessed the air pollution response in Ontario, Canada, during the COVID-19 emergency. Benmarhnia (2020) discussed the methodological challenges and opportunities for the link between air pollution and COVID-19, focusing on the role of air pollution as an influence modifier in the temporal and spatial variability of disease transmission and changes in the severity of symptoms and mortality. The indirect impact of interventions to control the human-tohuman transmission of new coronary pneumonia on air pollution and population health was also considered. Xiang et al. (2020) assessed the impact of COVID-19 on trafficrelated air pollution in cities in the northwestern United States and used a multiple autoregressive model to make comprehensive adjustments to weather. Cole et al. (2020a) used a two-step method to quantify the impact of Wuhan's new crown pneumonia epidemic prevention and control on the concentration of four air pollutants. First, machine learning is used to eliminate the confounding effects of weather conditions on pollution levels. Second, a new enhanced integrated control method is used to estimate the impact of the blockade on weather normalization pollution relative to a group of cities that are not blocked. Urrutia-Pereira et al. (2020) compiled environmental data released by multiple data centers and discussed the impact of the new crown pneumonia epidemic on environmental pollution. Azuma et al. (2020) conducted a longitudinal cohort study on the potential relationship between the spread of COVID-19 infection and climate or ambient air pollution in 28 regions of Japan. Son et al. (2020) investigated the impact of COVID-19 
mitigation measures on air pollution levels in ten US states and the District of Columbia and subsequent decline in mortality. Frank et al. (2020) evaluated the impact of ozone on crops during COVID-19 through improved models and methods and believed that the unprecedented and unexpected drop in air pollutant emissions may lead to a decrease in seasonal ozone concentration and have a positive impact on crop yields. Through the analysis of short-term and long-term drivers of carbon emissions, Wang and Wang (2020b) discussed how to prevent the retaliatory growth of carbon emissions after COVID-19.

Through literature review, we found that the current research had the following gaps. Although the relationship between COVID-19 and air pollutants has been discussed in many existing literatures, the current studies have focused more on the linear impact of the epidemic on air pollutants. The existing literatures involved many applications of linear models but only considered the direct effects between variables. There were few studies on the indirect influence of a factor on the relationship between variables. Thus, the queries were raised: Is there a nonlinear relationship between COVID19 and air pollutants? Is there a factor that has significant impact on the relationship between these two variables?

In order to cover the abovementioned gaps, this article had the following contributions. First, this article provided a new research perspective, based on the threshold panel regression model, to explore the nonlinear relationship between COVID19-confirmed cases and air pollution in the top ten cities in China's GDP. Second, a new variable "temperature" was added to observe the indirect influence of this factor on the relationship between COVID-19 and air pollutants.

Regarding remains of this paper, "Research methods and data sources" section introduced the research methods and data sources. "Empirical results and discussion" section analyzed the data calculation results. "Conclusion" section drew conclusions and put forward some specific questions and recommendations for public policy makers to consider when discussing COVID-19 and environmental issues.

\section{Research methods and data sources}

\section{Single threshold regression model}

Threshold panel regression model was usually used to study the nonlinear relationship between multiple variables (Wang and Wang 2021a). This paper selected the Hansen threshold regression model (Hansen 1999) for econometric analysis. The research objects were the top ten cities in China's GDP. It mainly explored whether there is a nonlinear relationship between confirmed cases and air pollutants at different stages of threshold variables.

\section{Model construction}

$y_{i, t}=\beta_{0} Z_{i t}+\beta_{1} R_{i t} I\left(q_{i t} \leq \gamma\right)+\beta_{2} R_{i t} I\left(q_{i t}>\gamma\right)+\mu_{i}+\varepsilon_{i, t}$

where $i$ represents the city and $t$ represents the date. $y_{i, t}$ is the indicator value of a certain air pollutant in city $i$ at time $t$, which is the explained variable. $q_{i t}$ is the threshold variable. $R_{i t}$ is the core explanatory variable affected by the threshold variable, that is, the number of confirmed cases in city $i$ at time t. $Z_{i t}$ is the variable that has significant impact on the air pollutant except for confirmed cases. $\beta_{0}, \beta_{1}$ and $\beta_{2}$ are corresponding coefficients, and $\gamma$ is specific threshold value. I $(\cdot)$ is an indicative function, and the value in the corresponding brackets is 1 when the condition is satisfied and 0 when the condition is not satisfied. $\mu_{i}$ is the individual effect, and $\varepsilon_{i, t}$ is random interference term. Equation (1) can also be expressed as follows:

$$
\begin{gathered}
y_{i, t}=\beta_{0} Z_{i t}+\beta R_{i t}\left(q_{i t}, \gamma\right)+\mu_{i}+\varepsilon_{i, t} \\
R_{i t}\left(q_{i t}, \gamma\right)=\left\{\begin{array}{c}
R_{i t} I\left(q_{i t} \leq \gamma\right) \\
R_{i t} I\left(q_{i t}>\gamma\right)
\end{array}\right.
\end{gathered}
$$

\section{Model estimation and testing}

Hansen believed that three issues need to be paid attention to. (1) Estimate the threshold value. (2) Test whether the threshold effect exists. (3) Test whether the threshold value is equal to the true value.

\section{Estimate the threshold value.}

Hansen proposed to use the two-stage least squares method to estimate the threshold panel model. Calculate the corresponding residual sum of squares for given threshold value, and then the threshold corresponding to the minimum value of all residual square sums is the threshold estimated value. The individual effect needs to be eliminated before model estimation (Wang and Wang 2021b). All cross sections of Eq. (1) are averaged within the group to obtain Eq. (3).

$y_{i, t}^{*}=\beta_{0} Z_{i, t}^{*}+\beta_{1} R_{i, t}^{*} I\left(q_{i t} \leq \gamma\right)+\beta_{2} R_{i, t}^{*} I\left(q_{i t}>\gamma\right)+\varepsilon_{i, t}^{*}$

The observations are stacked, and they are expressed as:

$y^{*}=R^{*}(\gamma) \beta+\varepsilon^{*}$

Therefore, for a given threshold value $\gamma$, the least square estimation of explanatory variable coefficients can be expressed as:

$\widehat{\beta}(\gamma)=\left\{R^{*}(\gamma)^{\prime} R^{*}(\gamma)\right\}^{-1}\left\{R^{*}(\gamma)^{\prime} y^{*}\right\}$ 
The residual sum of squares is:

$$
\mathrm{S}(\gamma)=\widehat{\varepsilon}^{*}(\gamma)^{\prime} \widehat{\varepsilon}^{*}(\gamma)
$$

The least squares estimate of the threshold $\gamma$ is $\widehat{\gamma}=\arg$ $\min \operatorname{SSE}(\gamma)$. The $\arg \min$ function represents the set of $\gamma$ corresponding to when the residual sum of squares is the smallest. The estimated residual vector is $\widehat{\varepsilon}^{*}$ $=\widehat{\varepsilon}^{*}(\widehat{\gamma})=y^{*}-R^{*}(\gamma) \beta$, the estimated residual variance is $\widehat{\sigma}^{2}$ $=\varepsilon^{*^{\prime}} \varepsilon^{*} / N(T-1)=S(\widehat{\sigma}) / N(T-1)$, and estimated regression coefficient is $\widehat{\beta}=\widehat{\beta}(\widehat{\gamma}) . T$ is the time span, and $N$ is the number of sample cities.

\section{Test whether the threshold effect exists.}

Hansen proposed the Bootstrap method with consistent heteroscedasticity to obtain its asymptotic distribution and then constructed the $P$ value and proved that the $P$ value constructed by this method is gradual and effective.

The null hypothesis is that there is no threshold effect:

$H_{0}: \beta_{1}=\beta_{2}$

The alternative hypothesis is:

$H_{1}: \beta_{1} \neq \beta_{2}$

The F statistic for testing the null hypothesis is:

$F_{1}=\frac{S_{0}-S_{1}(\hat{\gamma})}{\widehat{\sigma}^{2}}$

where $S_{0}$ and $S_{1}$ are the sum of squared residuals under the null hypothesis and alternative hypothesis, respectively. If the $P$ value is less than the expected critical value, the null hypothesis $H_{0}$ is rejected; that is, there is a threshold effect; otherwise, the null hypothesis is accepted, and the model is a linear model.

\section{(3) Test whether the threshold value is equal to the true value.}

Hansen pointed out that the best way to construct a confidence interval for $\gamma$ is to construct a "non-rejection domain" using likelihood ratio statistics. The null hypothesis is that the threshold value is equal to the true value, which can be expressed as $H_{0}: \gamma=\gamma_{0}$. The likelihood ratio statistic is

$L R(\gamma)=\frac{S_{1}(\gamma)-S_{1}(\widehat{\gamma})}{\widehat{\sigma}^{2}}$

Among them, $S_{1}(\gamma)$ and $S_{1}(\widehat{\gamma})$ are the sum of squared residuals of the null hypothesis and the alternative hypothesis, respectively. Hansen proved that $L R(\gamma)$ obeys a normal distribution. Assuming that $\mathrm{C}(\alpha)$ is the nonrejection interval, $\mathrm{C}(\alpha)=-2 \ln (1-\sqrt{1-\alpha}), \alpha$ is the significance level, and the effective asymptotic confidence interval is estimated accordingly. If $L R(\gamma)>\mathrm{C}(\alpha)$, reject the null hypothesis, and the threshold estimate is meaningless when the significance level is $\alpha$. Otherwise, accept the null hypothesis; that is, when the significance level is $\alpha$, the threshold estimate is meaningful.

\section{Multi-threshold regression model}

When it is determined that there is a threshold effect, it is necessary to continue to test whether there are two or more thresholds in the model. This article introduces the double threshold model, which is constructed as follows:

$$
\begin{aligned}
y_{i, t}= & \beta_{0} Z_{i t}+\beta_{1} R_{i t} I\left(q_{i t} \leq \gamma_{1}\right)+\beta_{2} R_{i t} I\left(\gamma_{1}<q_{i t}<\gamma_{2}\right) \\
& +\beta_{3} R_{i t} I\left(q_{i t} \geq \gamma_{2}\right)+\mu_{i}+\varepsilon_{i, t}
\end{aligned}
$$

where $\gamma_{1}$ and $\gamma_{2}$ are two threshold values and the corresponding coefficients are $\beta_{1}, \beta_{2}$ and $\beta_{3}$. When we know the first threshold estimated value $\widehat{\gamma_{1}}$, search for the second threshold $\gamma_{2}$. We arrive at:

$$
S_{2}^{\gamma}\left(\gamma_{2}\right)= \begin{cases}S\left(\widehat{(1}, \gamma_{2}\right) & \text { 若 } \widehat{\gamma_{1}}<\gamma_{2} \\ S\left(\gamma_{2}, \widehat{\gamma_{1}}\right) & \text { 若 } \gamma_{2}<\widehat{\gamma_{1}}\end{cases}
$$

The second threshold estimated value is $\widehat{\gamma}_{2}^{\gamma}=\arg \min$ $S_{2}^{\gamma}\left(\gamma_{2}\right)$. Next, we constructed the $F_{2}$ statistic to test whether the single threshold or the double threshold is more significant.
Table 1 Variable description and data sources

\begin{tabular}{lllll}
\hline Variable type & Variable name & Abbreviation & Unit & Data sources \\
\hline Explanatory variable & Confirmed cases & conf & person & NHC (2020) \\
Explained variable 1 & $\mathrm{NO} 2$ & $\mathrm{NO} 2$ & $\mu \mathrm{g} / \mathrm{m}^{3}$ & China Air Quality (2020) \\
Explained variable 2 & $\mathrm{PM} 2.5$ & $\mathrm{PM} 2.5$ & $\mu \mathrm{g} / \mathrm{m}^{3}$ & \\
Control variable & $\mathrm{PM} 10$ & $\mathrm{PM} 10$ & $\mu \mathrm{g} / \mathrm{m}^{3}$ & \\
Control variable & $\mathrm{SO} 2$ & $\mathrm{SO} 2$ & $\mu \mathrm{g} / \mathrm{m}^{3}$ & \\
Threshold variable & temperature & temp & ${ }^{\circ} \mathrm{C}$ & Temperature data (2020) \\
\hline
\end{tabular}


Table 2 Descriptive analysis of variables

\begin{tabular}{lllllllll}
\hline Variables & Obs & Mean & Sd & Min & Q1 & Q2 & Q3 & Max \\
\hline conf & 840 & 4966.307 & 14000 & 2.000 & 166.000 & 362.000 & 559.500 & 50000 \\
NO2 & 840 & 30.218 & 13.940 & 5.000 & 20.000 & 28.000 & 38.000 & 98.000 \\
PM2.5 & 840 & 33.935 & 21.178 & 3.000 & 21.000 & 30.000 & 43.000 & 207.000 \\
PM10 & 840 & 51.094 & 25.727 & 6.000 & 32.500 & 47.000 & 65.500 & 154.000 \\
SO2 & 840 & 6.116 & 2.291 & 2.000 & 5.000 & 6.000 & 7.000 & 17.000 \\
temp & 840 & 13.703 & 5.759 & -4.500 & 10.000 & 13.750 & 18.000 & 27.500 \\
\hline
\end{tabular}

Obs is the observed value, Mean is the average, $\mathrm{Sd}$ is the standard deviation, Min is the minimum, Q1 is the first quartile, Q2 is the median, Q3 is the third quartile, and Max is the maximum

$F_{2}=\frac{S_{1}\left(\widehat{\gamma}_{1}\right)-S_{2}^{\gamma}\left({\widehat{\gamma_{2}}}^{\gamma}\right)}{\widehat{\sigma}^{2}}$

It is an approximate likelihood ratio test. Wang and Wang (2020a) also agree with this point. If the value of $F_{2}$ is larger, then we reject the null hypothesis; that is, the double threshold effect is more significant.

\section{Variable selection and data source}

On January 30, 2020, the World Health Organization announced that COVID-19 was listed as a public health emergency of international concern. Therefore, the data in this article was for the time span of January 30 to April 22 (84 days). This article used the top ten cities in China's GDP ranking (Shanghai, Beijing, Shenzhen, Guangzhou, Chongqing, Suzhou, Wuhan, Chengdu, Hangzhou, Tianjin) from January 30 to April 22,
2020, as a panel data sample to explore the nonlinear relationship between confirmed cases and air pollution. We collected daily air pollution indicators data, temperature data, and the number of confirmed cases in 10 cities during the study period. We chose temperature as the threshold variable, the number of confirmed cases as the explanatory variable, $\mathrm{NO} 2$ and PM2.5 as the explained variables, and other air pollution indicators as the control variables. The variable description and data sources are shown in Table 1.

\section{Empirical results and discussion}

\section{Data descriptive analysis}

Based on the collected data, this article selected January 30 to April 22, 2020, as the sample interval, and the cross section was the top 10 cities in China's GDP. Each variable contained 840 pieces of data. The

Table 3 Unit root test of panel data

\begin{tabular}{|c|c|c|c|c|c|c|c|}
\hline Variables & & conf & $\mathrm{NO} 2$ & PM2.5 & PM10 & $\mathrm{SO} 2$ & temp \\
\hline \multicolumn{2}{|l|}{ Testing method } & \multicolumn{6}{|c|}{ Augmented Dickey-Fuller (ADF) } \\
\hline \multirow[t]{3}{*}{ At level } & t-Statistic & -1.9673 & -6.9808 & -13.6551 & -14.3751 & -7.2565 & -6.9085 \\
\hline & Prob. & 0.3015 & 0.0000 & 0.0000 & 0.0000 & 0.0000 & 0.0000 \\
\hline & Stability & No & Yes & Yes & Yes & Yes & Yes \\
\hline \multirow[t]{3}{*}{ At 1 st difference } & t-Statistic & -27.9134 & -18.3193 & -18.3145 & -11.5258 & -18.6071 & -20.4174 \\
\hline & Prob. & 0.0000 & 0.0000 & 0.0000 & 0.0000 & 0.0000 & 0.0000 \\
\hline & Stability & Yes & Yes & Yes & Yes & Yes & Yes \\
\hline \multicolumn{2}{|l|}{ Testing method } & \multicolumn{6}{|c|}{ Phillips-Perron (PP) } \\
\hline \multirow[t]{3}{*}{ At level } & t-Statistic & -2.2688 & -14.376 & -13.7901 & -14.6036 & -15.0988 & -6.9085 \\
\hline & Prob. & 0.1825 & 0.0000 & 0.0000 & 0.0000 & 0.0000 & 0.0000 \\
\hline & Stability & No & Yes & Yes & Yes & Yes & Yes \\
\hline \multirow[t]{3}{*}{ At 1st difference } & t-Statistic & -28.1026 & -115.212 & -98.6298 & -127.563 & -126.491 & -37.8955 \\
\hline & Prob. & 0.0000 & 0.0001 & 0.0001 & 0.0001 & 0.0001 & 0 \\
\hline & Stability & Yes & Yes & Yes & Yes & Yes & Yes \\
\hline
\end{tabular}


Table 4 Test results of threshold effect

\begin{tabular}{|c|c|c|c|c|c|c|}
\hline \multirow[t]{2}{*}{ Model } & \multirow[t]{2}{*}{$F$-value } & \multirow[t]{2}{*}{$P$-value } & \multirow{2}{*}{$\begin{array}{l}\text { Number } \\
\text { of Bootstrap }\end{array}$} & \multicolumn{3}{|c|}{ Critical value } \\
\hline & & & & $1 \%$ & $5 \%$ & $10 \%$ \\
\hline Single threshold & $18.451 *$ & 0.062 & 500 & 25.736 & 19.698 & 13.827 \\
\hline Double threshold & 0.016 & 0.874 & 500 & 2.295 & 1.886 & 1.426 \\
\hline Triple threshold & 2.256 & 0.290 & 300 & 6.074 & 4.864 & 4.090 \\
\hline
\end{tabular}

$* * *, * *$, and $*$ represent significance at the significance level of $1 \%, 5 \%$, and $10 \%$ respectively variables were confirmed cases (conf), NO2, PM2.5, $\mathrm{PM} 10, \mathrm{SO} 2$, and temperature (temp). The descriptive statistical results of the sample data are shown in Table 2. It can be seen that the standard deviation of confirmed cases in each city was relatively large, indicating that the spread of the epidemic in each city varied greatly. In addition, the degree of air pollution and temperature varied among cities.

\section{Unit root test}

Before constructing the model, it was necessary to test the stationarity of each variable, which is the unit root test of the variable. Only when each variable has passed the stationarity test can the next step be tested. The common unit root test methods were ADF test and PP test. This paper used these two testing methods to test the stationarity of six variables. According to whether the indicators have passed the $1 \%$ significance level as the test standard, the results are shown in Table 3. The results showed that all variables passed the tests of $\mathrm{ADF}$ and $\mathrm{PP}$ at 1 st difference, indicating that these variables are stable. Therefore, the data can be tested for the next step of the panel threshold effect.

\section{Panel threshold effect test and threshold value estimation}

\section{NO2 as the explained variable}

First, the temperature indicator was used as threshold variable to investigate the relationship between confirmed cases and

Table 5 Estimated threshold value

\begin{tabular}{lll}
\hline Model & Estimated threshold value & 95\% confidence interval \\
\hline Single threshold & $19.5^{*}$ & {$[17.5,20.0]$} \\
Double threshold & 21.5 & {$[21.5,22.5]$} \\
& 19.5 & {$[17.5,20.0]$} \\
Triple threshold & 10.0 & {$[4.0,21.5]$}
\end{tabular}

$* * *, * *$, and $*$ represent significance at the significance level of $1 \%, 5 \%$, and $10 \%$ respectively the concentration of NO2 in the air. Before regressing the threshold model, first check whether the threshold effect exists. We used the Bootstrap method proposed by Hansen to test the threshold variables. We judged the threshold effect of confirmed cases on $\mathrm{NO} 2$ concentration according to the tested $\mathrm{F}$ statistic and $\mathrm{P}$ value, and test whether there was a nonlinear relationship and the number of thresholds. The results are shown in Table 4. It can be seen that the F statistic in the single threshold model was 18.451 , and the $P$ value was 0.062 . The null hypothesis was rejected at the $10 \%$ significance level, indicating that there was a single threshold value.

According to the above threshold effect test, a single threshold regression calculation was performed on the model. The estimated threshold is shown in Table 5 and Fig. 1. We can see that the threshold estimate obtained by the single threshold regression model was 19.5 . The $95 \%$ confidence interval was $[17.5,20.0]$, the confidence interval was narrow, and the threshold estimate was credible.

In this paper, without adding threshold variables, the ordinary panel model was regressed, and the fixed effect model was used to obtain parameter estimates. We compared the regression results of the threshold effect model and the fixed effect model, as shown in Table 6.

The results showed that in the fixed effect model, the confirmed case had a negative effect on the $\mathrm{NO} 2$ concentration with a coefficient of -0.00009 . PM2.5 and NO2 also showed a

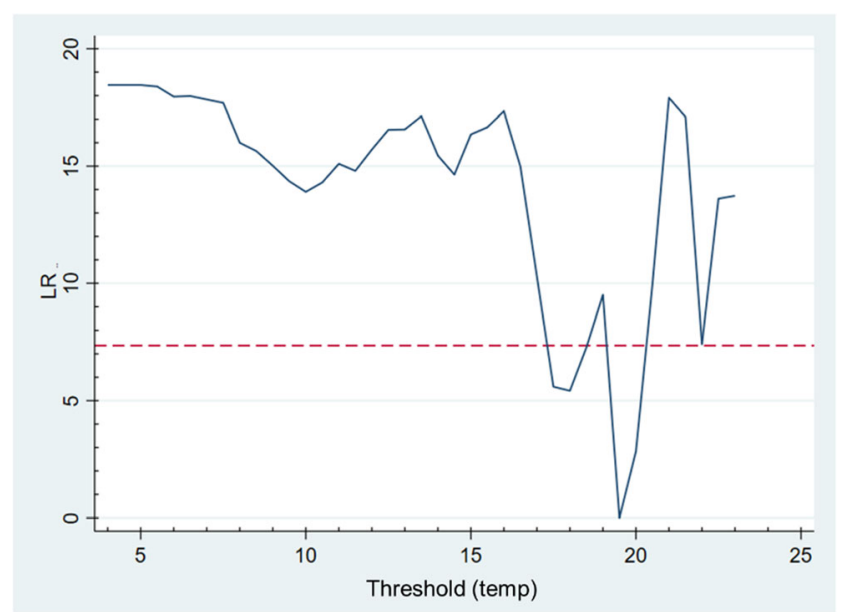

Fig. 1 Single threshold estimate 
Table 6 Regression results of threshold model and fixed effect model

\begin{tabular}{lll}
\hline Variables & Threshold model (NO2) & Fixed effect model (NO2) \\
\hline conf & -- & -0.00009 \\
& & $(0.00007)$ \\
conf (temp $\leq 19.5)$ & $-0.00005(0.00007)$ & -- \\
conf (temp $>19.5)$ & $-0.00038 * * *(0.00010)$ & -- \\
PM2.5 & $-0.03434(0.02742)$ & $-0.02992(0.02766)$ \\
PM10 & $0.22201 * * *(0.02610)$ & $0.22710 * * *(0.02631)$ \\
SO2 & $2.89137 * * *(0.24411)$ & $2.61465 * * *(0.23585)$ \\
Constant & $2.81764 * *(1.27499)$ & $4.10198 * * *(1.24366)$ \\
\hline
\end{tabular}

$* * *, * *$, and $*$ represent significance at the significance level of $1 \%, 5 \%$, and $10 \%$ respectively. The content in parentheses is the standard deviation.

negative correlation with a coefficient of -0.02992 . But the effect of these two variables was not significant. The positive effects of PM10 and SO2 were significant.

In the threshold regression model, the influence of explanatory variables on $\mathrm{NO} 2$ concentration was also negatively correlated. When the temperature crossed the threshold, the negative effect of confirmed cases on $\mathrm{NO} 2$ was strengthened. When the temperature was lower than $19.5^{\circ} \mathrm{C}$, the number of confirmed cases increased by $1 \%$, and the $\mathrm{NO} 2$ concentration decreased by about $0.005 \%$. When the temperature was higher than $19.5^{\circ} \mathrm{C}$, the number of confirmed cases increased by $1 \%$, and the NO2 concentration decreased by approximately $0.038 \%$. And when the temperature crossed the threshold, the significance of the negative correlation between variables was more obvious. Some scholars put forward many suggestions to protect the environment. Hua et al. (2021) believed that waste recycling behavior is a newly developing proenvironment behavior. More targeted measures are needed to reduce air pollution and protect the environment.

\section{PM2.5 as the explained variable}

Second, this article used temperature indicators as threshold variables to investigate the relationship between confirmed cases and PM2.5 concentrations in the air. We used the Bootstrap method proposed by Hansen to check whether the
Table 8 Estimated threshold value

\begin{tabular}{lll}
\hline Model & $\begin{array}{l}\text { Estimated } \\
\text { threshold value }\end{array}$ & 95\% confidence interval \\
\hline Single threshold & $20.5^{*}$ & {$[4.0,23.0]$} \\
Double threshold & 21.5 & {$[5.5,22.5]$} \\
& 20.5 & {$[4.0,22.5]$} \\
Triple threshold & 14.0 & {$[4.0,22.5]$}
\end{tabular}

$* * *, * *$, and $*$ represent significance at the significance level of $1 \%, 5 \%$, and $10 \%$ respectively

threshold effect exists. The test results are shown in Table 7. It can be seen that the F statistic in the single threshold model was 4.358 , and the $P$ value was 0.066 . The null hypothesis was rejected at the $10 \%$ significance level, indicating that there was a single threshold value. Based on the threshold effect test, a single threshold regression was performed on the model. The threshold estimated value is shown in Table 8 . The threshold estimated value obtained by the single threshold regression model was 20.5 .

This paper used a fixed effect model to regress the ordinary panel model without adding threshold variables. We compared the parameter estimates of the threshold effect model and the fixed effect model, as shown in Table 9.

The results showed that in the fixed effect model, the impact of confirmed cases on PM2.5 concentration was negative, and it was highly significant at the $1 \%$ significance level. The calculated coefficient was -0.00035 .

In the threshold regression model, the impact of confirmed cases on PM2.5 concentration also showed a highly significant negative correlation. When the temperature crossed the threshold, the negative effect of confirmed cases on PM2.5 was strengthened. When the temperature was lower than $20.5^{\circ} \mathrm{C}$, the number of confirmed cases increased by $1 \%$, and the PM2.5 concentration decreased by $0.033 \%$. When the temperature was higher than $20.5^{\circ} \mathrm{C}$, the number of confirmed cases increased by $1 \%$, and the PM2.5 concentration decreased by $0.061 \%$.

In summary, confirmed cases had a nonlinear effect on the $\mathrm{NO}_{2}$ concentration and PM2.5 concentration in the air, and both showed a negative correlation. This result was not difficult to understand. In order to control the spread of the epidemic, the country had adopted an isolation policy of
Table 7 Test results of threshold effect

\begin{tabular}{lllllll}
\hline Model & F-value & P-value & $\begin{array}{l}\text { Number } \\
\text { of Bootstrap }\end{array}$ & & \multicolumn{2}{l}{ Critical value } \\
\cline { 5 - 7 } & & & & $1 \%$ & $5 \%$ & $10 \%$ \\
\hline Single threshold & $4.358^{*}$ & 0.066 & 500 & 12.447 & 5.157 & 3.551 \\
Double threshold & 1.577 & 0.140 & 500 & 5.143 & 2.870 & 1.947 \\
Triple threshold & 1.744 & 0.310 & 300 & 28.841 & 13.840 & 8.607 \\
\hline
\end{tabular}

$* * *, * *$, and $*$ represent significance at the significance level of $1 \%, 5 \%$, and $10 \%$ respectively 
Table 9 Regression results of threshold model and fixed effect model Variables Threshold model PM2.5 Fixed effect model PM2.5

\begin{tabular}{lll}
\hline conf & - & $-0.00035 * * *(0.00010)$ \\
conf $($ temp $\leq 20.5)$ & $-0.00033 * * *(0.00010)$ & -- \\
conf (temp $>20.5)$ & $-0.00061 * * *(0.00016)$ & -- \\
PM10 & $0.68352 * * *(0.04037)$ & $-0.05257(0.04859)$ \\
SO2 & $-0.84462 * *(0.36089)$ & $0.68311 * * *(0.02665)$ \\
NO2 & $-0.05238(0.15104)$ & $-0.83257 * *(0.33609)$ \\
Constant & $7.49732 * *(4.55919)$ & $7.45232 * * *(1.63781)$ \\
\hline
\end{tabular}

$* * *, * *$, and $*$ represent significance at the significance level of $1 \%, 5 \%$, and $10 \%$ respectively. The content in parentheses is the standard deviation.

"blocking the city." The economic development of various cities was subject to certain restrictions, and the reduction of economic activities in various industries had led to a reduction in the emission of air pollutants. Furthermore, we found that when the threshold was crossed, the negative effects of confirmed cases on the concentration of $\mathrm{NO}_{2}$ and PM2.5 in the air were significantly enhanced. Making reasonable policies was important to China's environmental protection (Liu and Dong 2021).

\section{Conclusion}

This paper developed panel threshold regression approach to investigate the impact of COVID-19-confirmed cases on air pollution in the China's top ten cities. The computing result showed that there is a nonlinear relationship between COVID19 and air pollutants, and the temperature variable has a significant impact on the relationship between these two variables. The confirmed cases had a nonlinear effect on the $\mathrm{NO}_{2}$ concentration in the air, showing a negative correlation. When the temperature crossed the threshold, the negative effect of confirmed cases on $\mathrm{NO}_{2}$ was strengthened, and the significance of the negative correlation between the variables was more obvious. The impact of the confirmed disease on the PM2.5 concentration in the air also showed a highly significant negative correlation. When the temperature crossed the threshold, the negative effect of confirmed cases on PM2.5 was strengthened.

This research helps us clarify the relationship between human production and operation activities caused by COVID-19 and air pollution and provides richer information for future environmental policy design. Effectively controlling the epidemic and reducing the emission of air pollutants are issues that need to be focused on. Public policy makers need to prevent the retaliatory rebound of air pollutants based on the resumption of work and production in society. This article suggests that in the short term, the stability of various industries can be controlled through corresponding incentives or subsidies, while in the long term, it is necessary to incorporate urban emission reduction technologies into local assessment targets to improve efficiency; include areas with inadequate implementation of comprehensive air pollution control tasks, prominent environmental problems, and obvious deterioration of ambient air quality into the central ecological environment protection inspection category; implement hierarchical and classified management and control of corporate performance, strengthen joint prevention and control, and continue to promote clean energy; and adhere to the problem orientation, compact the responsibilities of departments and localities, and increase assistance to prevent the rebound of heavy pollution.

This paper is conducted from a new perspective, considering the nonlinearity, and adding the temperature variable, which provides a methodological reference for studying the nonlinear relationship between the epidemic situation and different pollution factors. In addition, this article also expands the scope of application of the threshold model, enriching the quantitative analysis and research of epidemics and pollutants.

Author contribution Qiang Wang: Conceptualization, methodology, software, data curation, writing - original draft preparation, supervision, and writing - reviewing and editing. Xiaowei Wang: Methodology, software, investigation, writing - original draft, and writing - reviewing and editing

Funding This work is funded by National Natural Science Foundation of China (Grant No. 71874203), Humanities and Social Science Fund of Ministry of Education of China (Grant No.18YJA790081), and Natural Science Foundation of Shandong Province, China (Grant No. ZR2018MG016).

Data Availability The datasets used and/or analyzed during the current study are available from the corresponding author on reasonable request.

\section{Declarations}

Ethics approval and consent to participate Not applicable

Consent for publication Not applicable

Competing interests The authors declare no competing interests.

\section{References}

Adams MD (2020) Air pollution in Ontario, Canada during the COVID19 State of Emergency. Sci Total Environ 742:140516

Azuma K, Kagi N, Kim H, Hayashi M (2020) Impact of climate and ambient air pollution on the epidemic growth during COVID-19 outbreak in Japan. Environ Res 190:110042 
Benmarhnia T (2020) Linkages between air pollution and the health burden from COVID-19: methodological challenges and opportunities. Am J Epidemiol 189:1238-1243

Berman JD, Ebisu K (2020) Changes in U.S. air pollution during the COVID-19 pandemic. Sci Total Environ 739:139864

Chen K, Wang M, Huang C, Kinney PL, Anastas PT (2020) Air pollution reduction and mortality benefit during the COVID-19 outbreak in China The Lancet Planetary. Health 4:e210-e212

China Air Quality (2020) Air quality historical data. https://www. aqistudy.cn/historydata/ (in Chinese)

Cole MA, Elliott RJR, Liu B (2020a) The impact of the Wuhan COVID19 lockdown on air pollution and health: a machine learning and augmented synthetic control approach. Environ Resour Econ 76: $553-580$

Cole MA, Ozgen C, Strobl E (2020b) Air Pollution Exposure and Covid19. Department of Economics, University of Birmingham, Discussion Papers 20-13

Comunian S, Dongo D, Milani C, Palestini P (2020) Air pollution and COVID-19: the role of particulate matter in the spread and increase of COVID-19's morbidity and mortality. Int J Environ Res Public Health 17:4487

Contini D, Costabile F (2020) Does air pollution influence COVID-19 outbreaks? Atmosphere 11:377

Copat C, Cristaldi A, Fiore M, Grasso A, Zuccarello P, Signorelli SS, Conti GO, Ferrante M (2020) The role of air pollution (PM and NO2) in COVID-19 spread and lethality: a systematic review. Environ Res 191:110129

Domingo JL, Marquès M, Rovira J (2020) Influence of airborne transmission of SARS-CoV-2 on COVID-19 pandemic. a review. Environ Res 188:109861

Dong F, Liu Y (2020) Policy evolution and effect evaluation of newenergy vehicle industry in China. Res Policy 67:101655

Fattorini D, Regoli F (2020) Role of the chronic air pollution levels in the Covid-19 outbreak risk in Italy. Environ Pollut 264:114732

Frank D et al (2020) Lower air pollution during COVID-19 lock-down: improving models and methods estimating ozone impacts on crops. Phil Trans R Soc A 378:20200188

Gupta A, Bherwani H, Gautam S (2020) Air pollution aggravating COVID-19 lethality? Exploration in Asian cities using statistical models. Environ Dev Sustain 23:6408-6417

Hansen BE (1999) Threshold effects in non-dynamic panels: estimation, testing, and inference. J Econ 93:345-368

He G, Pan Y, Tanaka T (2020) The short-term impacts of COVID-19 lockdown on urban air pollution in China. Nat Sustain 3:1005-1011

Hendryx M, Luo J (2020) COVID-19 prevalence and fatality rates in association with air pollution emission concentrations and emission sources. Environ Pollut 265:115126

Hua Y, Dong F, Goodman J (2021) How to leverage the role of social capital in pro-environmental behavior: a case study of residents' express waste recycling behavior in China. J Clean Prod 280: 124376

Le T, Wang Y, Liu L, Yang J, Yung YL, Li G, Seinfeld JH (2020) Unexpected air pollution with marked emission reductions during the COVID-19 outbreak in China. Science 369:702-706

Lin S, Wei D, Sun Y, Chen K, Yang L, Liu B, Huang Q, Paoliello MMB, $\mathrm{Li} \mathrm{H}, \mathrm{Wu} \mathrm{S}$ (2020) Region-specific air pollutants and meteorological parameters influence COVID-19: a study from mainland China. Ecotoxicol Environ Saf 204:111035

Liu Y, Dong F (2021) Haze pollution and corruption: a perspective of mediating and moderating roles. J Clean Prod 279:123550
Magazzino C, Mele M, Schneider N (2020) The relationship between air pollution and COVID-19-related deaths: an application to three French cities. Appl Energy 279:115835

NHC (2020) National Health Commission of the People's Republic of China. http://www.nhc.gov.cn/ (in Chinese)

Pei Z, Han G, Ma X, Su H, Gong W (2020) Response of major air pollutants to COVID-19 lockdowns in China. Sci Total Environ $743: 140879$

Saez M, Tobias A, Barceló MA (2020) Effects of long-term exposure to air pollutants on the spatial spread of COVID-19 in Catalonia, Spain. Environ Res 191:110177

Shehzad K, Sarfraz M, Shah SGM (2020) The impact of COVID-19 as a necessary evil on air pollution in India during the lockdown. Environ Pollut 266:115080

Singh V, Singh S, Biswal A, Kesarkar AP, Mor S, Ravindra K (2020) Diurnal and temporal changes in air pollution during COVID-19 strict lockdown over different regions of India. Environ Pollut 266:115368

Son J-Y, Fong KC, Heo S, Kim H, Lim CC, Bell ML (2020) Reductions in mortality resulting from reduced air pollution levels due to COVID-19 mitigation measures. Sci Total Environ 744:141012

Temperature data (2020) Temperature data. http://www.tianqihoubao. com/lishi/ (in Chinese)

Urrutia-Pereira M, Mello-da-Silva CA, Solé D (2020) COVID-19 and air pollution: a dangerous association? Allergol Immunopathol 48:496499

Wang Q, Wang L (2020a) Renewable energy consumption and economic growth in OECD countries: a nonlinear panel data analysis. Energy 207:118200

Wang Q, Wang S (2020b) Preventing carbon emission retaliatory rebound post-COVID-19 requires expanding free trade and improving energy efficiency. Sci Total Environ 746:141158

Wang Q, Wang L (2021a) How does trade openness impact carbon intensity? J Clean Prod 295:126370

Wang Q, Wang L (2021b) The nonlinear effects of population aging, industrial structure, and urbanization on carbon emissions: a panel threshold regression analysis of 137 countries. J Clean Prod 287: 125381

Wang P, Chen K, Zhu S, Wang P, Zhang H (2020) Severe air pollution events not avoided by reduced anthropogenic activities during COVID-19 outbreak Resources. Conserv Recycl 158:104814

Wu X, Nethery RC, Sabath MB, Braun D, Dominici F (2020) Exposure to air pollution and COVID-19 mortality in the United States: a nationwide cross-sectional study. Sci Adv 6:4049. https://doi.org/ $10.1101 / 2020.04 .05 .20054502$

Xiang J et al (2020) Impacts of the COVID-19 responses on traffic-related air pollution in a Northwestern US city. Sci Total Environ 747: 141325

Zhang Z, Xue T, Jin X (2020) Effects of meteorological conditions and air pollution on COVID-19 transmission: evidence from 219 Chinese cities. Sci Total Environ 741:140244

Zhu Y, Xie J, Huang F, Cao L (2020) Association between short-term exposure to air pollution and COVID-19 infection: evidence from China. Sci Total Environ 727:138704

Publisher's note Springer Nature remains neutral with regard to jurisdictional claims in published maps and institutional affiliations. 